Network Working Group

RFC \# 516

NIC \# 16683
Jonathan B. Postel

UCLA-NMC

May 18, 1973

\title{
LOST MESSAGE DETECTION
}

I have three suggestions for detecting the loss of messages by the communications subsystem. The first of these is perhaps the more powerful and simpler to implement since it uses no new concepts and has the power to retransmit the message detected as lost.

The first scheme:

If upon sending a message the host saved a copy of that message and waited until either:

a RFNM was returned, in which case everything is ok and the next message is processed.

a INCOMPLETE TRANSMISSION is returned, in which case the copy of the message is retransmitted (this could be a loop so put a finite upper bound on the number of times to retransmit the same message).

a DESTINATION DEAD is returned, in which case mark the destination down and require the exchange of reset commands before further communication is allowed.

something else is received indicating an error in the network or local IMP, in which case at least log the error, and probably close the conversation.

Following the above procedures either on a per host basis or a per link basis should prevent a lost message problem from developing.

The second scheme:

If on a per host basis, message numbers are included in the host to host header of messages, , and messages are delivered in order (this is currently the case in the network, except for priority messages so this proposal requires that each host either send everything as priority or nothing as priority) then each receiving host can detect a missing message by comparing the message number of the received message with the previously received message.

On exchanging resets the sequence numbers between that pair of 
hosts is set to zero.

Each time a message is sent the current send message number is entered into a field in the message header, and the current send message number is incremented (modulo $N$, say $N=256$ )

Each time a message is received the message number from the message is compared to the current receive message number and:

if the received message is the expected one then the message is acceptable and current receive message number is incremented (modulo N); if the received message is not the expected one then a message has been lost.

What to do when a missing message is detected, not clear, but at least can be logged and reported to the network control center. A missing message may not be fatal to an interactive conversation, but it is critical in a file transfer, thus I suggest that missing messages which are not recovered be cause to close the conversation.

The third scheme:

Host to host acknowledgements could be required. Such an acknowledgement scheme could be implemented similarly to the IMP to IMP scheme. This is a serious change to the current protocols so I will not elaborate on it here, feeling that deeper study will be necessary to fully specify a reasonable host to host acknowledgement strategy.

Of these three suggestions the first is the most immediately practical and implementable; in fact several hosts all ready do this. These schemes also are non-conflicting, they could be implemented and used simultaneously.

[ This RFC was put into machine readable form for entry ]

[ into the online RFC archives by Alex McKenzie with ]

[ support from GTE, formerly BBN Corp. 9/99] 